\title{
Silencing of long non-coding RNA ANRIL inhibits the development of multidrug resistance in gastric cancer cells
}

\author{
WEI-GUANG LAN ${ }^{1}$, DIAN-HONG XU ${ }^{1}$, CHEN XU $^{2}$, CHANG-LING DING ${ }^{3}$, FANG-LING NING $^{1}$, \\ YAN-LI ZHOU ${ }^{3}$, LONG-BO MA ${ }^{1}$, CHANG-MIN LIU ${ }^{1}$ and XIA HAN ${ }^{1}$ \\ ${ }^{1}$ Department of Oncology, Affiliated Hospital of Binzhou Medical University, Binzhou, Shandong; \\ ${ }^{2}$ The Second Department of Internal Medicine, People's Hospital of Kenli County, Kenli, Shandong; \\ ${ }^{3}$ Department of Pharmacy, Affiliated Hospital of Binzhou Medical University, Binzhou, Shandong, P.R. China
}

Received December 25, 2015; Accepted March 1, 2016

DOI: $10.3892 /$ or.2016.4771

\begin{abstract}
The development of multidrug resistance (MDR) is a crucial cause of therapy failure in gastric cancer, which results in disease recurrence and metastasis. Long non-coding RNAs (lncRNAs) have been proven to be critical in carcinogenesis and metastasis of gastric cancer. However, little is known about the roles of ANRIL (antisense non-coding RNA in the INK4 locus) in gastric cancer MDR. The aim of our study is to identify the biological function of ANRIL in gastric cancer MDR. In our results, ANRIL was highly expressed in gastric cancer tissues of cisplatin-resistant and 5-fluorouracil (5-FU)-resistant patients, and the same upregulation trends were observed in cisplatin-resistant cells (BGC823/DDP) and 5-FU-resistant cells (BGC823/5-FU). In addition, BGC823/DDP and BGC823/5-FU cells transfected with ANRIL siRNA and treated with cisplatin or 5-FU, respectively, exhibited significant lower survival rate, decreased invasion capability, and high percentage of apoptotic tumor cells. The influence of ANRIL knockdown on MDR was assessed by measuring $\mathrm{IC}_{50}$ of BGC823/DDP and BGC823/5-FU cells to cisplatin and 5-FU, the result showed that silencing ANRIL decreased the $\mathrm{IC}_{50}$ values in gastric cancer cells. Moreover, qRT-PCR and western blotting revealed that ANRIL knockdown decreased the expression of MDR1 and MRP1, both of which are MDR related genes; regression analysis showed that the expression of ANRIL positively correlated with the expression of MDR1 and MRP1, resprectively In summary, knockdown of lncRNA ANRIL in gastric cancer cells inhibits the development of MDR, suggesting an efficacious target for reversing MDR in gastric cancer therapy.
\end{abstract}

Correspondence to: Dr Dian-Hong Xu, Department of Oncology, Affiliated Hospital of Binzhou Medical University, 661 Yellow River Second Road, Binzhou, Shandong 256603, P.R. China E-mail: byfuhl_2015@163.com

Key words: long non-coding RNA, ANRIL, multidrug resistance, gastric cancer

\section{Introduction}

Gastric cancer is the fourth most common type of cancer and the second leading cause of cancer-related deaths in the world (1). Chemotherapy plays an important role in the treatment of gastric cancer both in adjuvant and advanced settings. It is known that MDR involves a large number of molecules and complex mechanisms. Classical drug-resistant molecules, such as multidrug resistance gene 1 (MDR1) (2) and multi-drug resistance protein (MRP1) (3), have been found to play important roles in mediating MDR in some gastric cancers. However, the efficacy of chemotherapy for gastric cancer is limited due to insensitivity and the development of MDR (4).

LncRNAs with lengths of more than $200 \mathrm{nt}$ are abundant in the human genome and have attracted increasing scientific interest (5). Despite being initially regarded as 'transcriptional noise', accumulating evidence has found that lncRNAs can manipulate local or global gene expression via transcriptional, post-transcriptional and epigenetic regulation (6). The IncRNAs that have been characterized are implicated in diverse physiological and pathological processes, such as $\mathrm{X}$-chromosome inactivation (7), stem cell pluripotency (8), development (9) and cancer metastasis (10). However, little is known about the function of IncRNAs in gastric cancer MDR.

INK4b-ARF-INK4a encodes 3 tumor-suppressor proteins, p15 (INK4b), p14 (ARF), and p16 (INK4a), and its transcription is a key requirement for replicative or oncogene-induced senescence and constitutes an important barrier for tumor growth (11). Long non-coding RNA ANRIL was identified by Pasmant et al (12), it is transcribed as a 3.8-kb lncRNA in the opposite direction from the INK4B-ARF-INK4A gene cluster (13). In a previous study, Aguilo et al reported that both polycomb repressive complex-1 (PRC1) and PRC2 interact with IncRNA ANRIL to form heterochromatin surrounding the INK4b-ARF-INK4a locus, leading to its repression (14). Pasmant et al also identified ANRIL as a modifier gene in neurofibromatosis type I, which may offer clues to the molecular pathogenesis of plexiform neurofibromas, particularly neurofibroma formation (15). In addition, Özgür et al showed that lncRNA ANRIL was upregulated during DNA damage-induced cell death in HeLa and caspase-3-deficient MCF-7 cells using bleomycin and $\gamma$-radiation to induce DNA 
damage (16). However, the role of ANRIL in gastric cancer MDR remains largely unexplored.

In the present study, we explored the role of IncRNA ANRIL in cisplatin-resistant and 5-FU-resistant gastric cancer and further investigated the biological function of ANRIL on BGC823/DDP and BGC823/5-FU cells. Furthermore, we evaluated the effects of silencing ANRIL on the expression of genes associated with multidrug resistant, including MDR1 and MRP1.

\section{Materials and methods}

Patient characteristics and clinicopathological features. A total of 83 patients with primary gastric cancer were enrolled in this study. The patients received cisplatin-based $(n=47)$ or 5-FU-based $(n=36)$ palliative chemotherapy without surgery. Obvious primary tumor shrinkage and reduction of malignant pleural effusions were considered effective treatment. Cisplatin-resistant or 5-FU-resistant cases were distinguished when primary tumor enlarged, pleural effusions increased or new metastasis occurred within 12 months, otherwise cisplatin-sensitive $(\mathrm{n}=11)$ and 5 -FU-sensitive $(\mathrm{n}=8)$ case was defined. The tumor tissue samples were collected from these patients. This study received approval from the Ethics Committee of Affiliated Hospital of Binzhou Medical University, and all patients provided written informed consent.

Cell lines and culture. Human gastric cancer cell lines BGC823 were purchased from the Beijing Zhongyuan Biotech (Beijing, China). The cells were grown at $37^{\circ} \mathrm{C}$ in a humidified incubator with $5 \% \mathrm{CO}_{2}$. Cisplatin and 5-FU were obtained from Sigma-Aldrich (St. Louis, MO, USA). The cisplatin-resistant BGC823/DDP cells were developed from the parental BGC823 cells that were subjected to persistent gradient exposure to cisplatin for about 12 months, through increasing cisplatin concentration from $0.05 \mathrm{mg} / \mathrm{ml}$ until the cells acquired resistance to $1 \mathrm{mg} / \mathrm{ml}$. The 5 -FU-resistant BGC823/5-FU cells were obtained in the same way.

siRNA and transfection. siRNA specially targeting ANRIL were purchased from GenePharma Biotech (Shanghai, China), the sequence was as follows: sense, 5'-GGGCCAGAGUCAA GAUUUAUU-3' and antisense, 5'-UAAAUCUUGACUCUGG CCCUU-3'. BGC823 control cells, BGC823/DDP and BGC823/5-FU cells were seeded in 6-well plates, after 24-h incubation, cells were transfected with either $50 \mathrm{nM}$ siRNAs targeting ANRIL (si-ANRIL) or scrambled negative controls (si-NC) using the Lipofectamine 2000 transfection reagent (Invitrogen) according to the instructions provided by the manufacturer.

Quantitative real-time PCR. Total RNA (500 ng) was used for cDNA synthesis by Superscript III First Strand synthesis (Invitrogen, USA). SYBR Premix Taq (BioRad, USA) was used for real-time PCR assays. Glyceraldehyde-3-phosphate dehydrogenase $(G A P D H)$ were detected and the mRNA levels were used as endogenous controls. Primer pairs used for quantitative RT-PCR were from Sigma-Aldrich and their sequences are: ANRIL sense, 5'-TTCGGCTGTGAATCCATC-3' and antisense, 5'-CCATACCATAGTGCGTTAG-3'; GAPDH sense, 5'-CTCACCGGATGCACCAATGTT-3' and antisense, 5'-CGCGTTGCTCACAATGTTCAT-3'; MDRI sense, 5'-TTG GCTGATGTTTGTGGGAAG-3' and antisense, 5'-CCAAAA ATGAGTAGCACGCCT-3'; and $M R P 1$ sense, 5'-GTGAATCG TGGCATCGACATA-3' and antisense, 5'-GCTTGGGACGGA AGGGAATC-3'.

Cytotoxicity assay. The cytotoxicity of gene transfection was determined by Cell Counting Kit-8 (CCK-8) assay. In 96-well plates, cells were seeded in 100- $\mu$ 1 PRMI-1640 medium supplemented with $10 \% \mathrm{FBS}$ at $5 \times 10^{4}$ cells/well. Then chemotherapeutic agents were added in normal growth medium supplemented with FBS. After $48 \mathrm{~h}$ incubation, $10 \mu \mathrm{l} \mathrm{CCK-8}$ was added and culture was continued for $1 \mathrm{~h}$ in humidified atmosphere containing $5 \% \mathrm{CO}_{2}$. Absorbances at $450 \mathrm{~nm}$ were measured by microplate reader (Bio-Tech Company). The relative drug resistance folds were analyzed by comparison with $\mathrm{IC}_{50}$.

Western blot assays and antibodies. Cell lysates were prepared in a buffer containing $100 \mathrm{mM} \mathrm{NaCl}, 10 \mathrm{mM}$ Tris- $\mathrm{HCl}(\mathrm{pH} 7.6)$, $1 \mathrm{mM}$ EDTA (pH 8.0), $1 \mu \mathrm{g} / \mathrm{ml}$ aprotinin, $100 \mu \mathrm{g} / \mathrm{ml}$ phenylmethylsulfonyl fluoride, and $1 \%(\mathrm{v} / \mathrm{v})$ NP-40. After protein quantitation using the Lowery protein assay, equal amounts of proteins were separated by SDS-PAGE and blotted onto nitrocellulose membranes by the semi-dry blotting method. The membranes were incubated with a dilution of primary antibody (anti-MDR1, sc-55510, 1:500 dilution; anti-MRP1, sc-365635, 1:500 dilution; anti-GAPDH, sc-25778, 1:1,000 dilution), overnight at $4^{\circ} \mathrm{C}$. The membrane was washed with TBST and incubated with a peroxidase-conjugated secondary antibody (1:2,000; Zhongshan Jinqiao Biotech, Beijing, China) for $1 \mathrm{~h}$. Specific antibody binding was detected using a chemiluminescence detection system (Pierce, Rockford, IL, USA), according to the manufacturer's recommendations.

Cell proliferation assays. Cell viability of gastric cancer cells transfected with si-ANRIL or si-NC was assessed by the 3-(4,5-dimethylthiazol-2-yl)-2,5-diphenyltetrazolium bromide (MTT) assay. Briefly, $48 \mathrm{~h}$ after transfection, $20 \mu 1 \mathrm{MTT}$ solution (Sigma-Aldrich) was added into the culture medium for $4 \mathrm{~h}$ incubation. Then, $150 \mu \mathrm{l}$ DMSO (Sigma-Aldrich) was added into each well to dissolve the crystals. The absorbance of each sample was recorded at $490 \mathrm{~nm}$. All experiments were performed in quadruplicate. For each treatment group, wells were assessed in triplicate.

Wound-healing assay. Approximately $5 \times 10^{4}$ cells were seeded in 24-well plates and cultured until 70-80\% confluent. Wounds were established using a $20-\mu \mathrm{l}$ pipette tip and the cells were allowed $24 \mathrm{~h}$ to migrate into the wounds. To assess the migration of the cells across the artificial wound, a total of five optical fields were randomly selected and analyzed using a Leica DMI 4000B inverted microscope with the Leica application suite software (Leica Microsystems).

Cell invasion assay. The invasive potential of cells was measured in 12-well Matrigel-coated invasion chambers (BD Biosciences, Bedford, MA, USA). The lower chambers were filled with $0.75 \mathrm{ml}$ of RPMI-1640 medium containing $10 \%$ fetal 
A

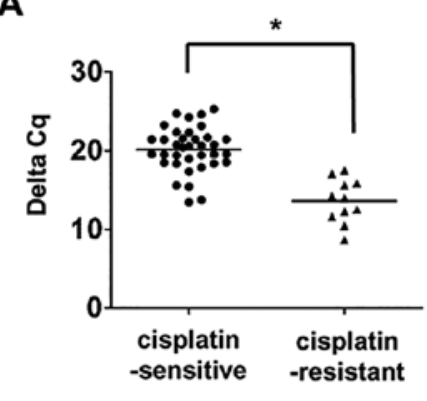

B

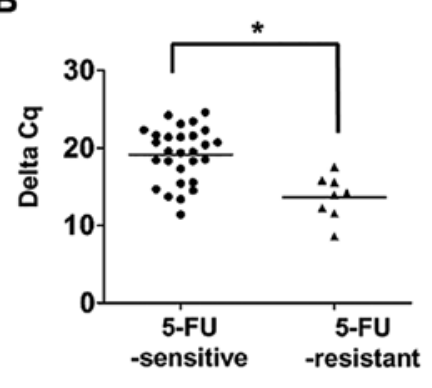

C

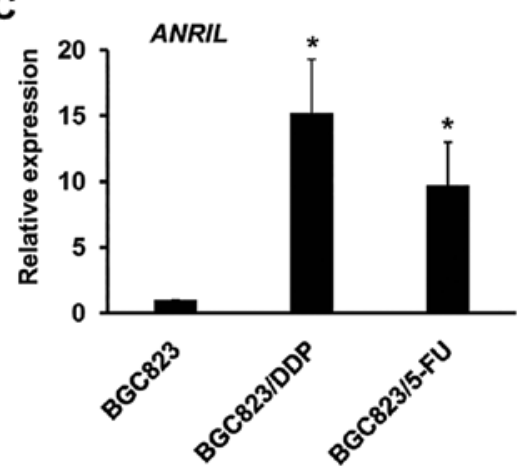

Figure 1. ANRIL is upregulated in cisplatin-resistant and 5-FU-resistant gastric cancer tissues and cells. (A) The levels of ANRIL expression in gastric cancer tissue of cisplatin-sensitive patients $(n=36)$ and cisplatin-resistant patients $(n=11)$. (B) The levels of ANRIL expression in gastric cancer tissue of 5-FU-sensitive patients $(n=28)$ and 5-FU-resistant patients $(n=8)$. (C) The mRNA levels of ANRIL in the normal BGC823 cells, BGC823/DDP cells and BGC823/5-FU cells. Error bars indicate means \pm standard errors of the mean. ${ }^{*} \mathrm{P}<0.05$ vs. control.

bovine serum (FBS). A cell suspension of $2.5 \times 10^{4}$ in $0.5 \mathrm{ml}$ RPMI-1640 medium was added into each well of the upper chamber. After the cells were incubated for $20 \mathrm{~h}$ at $37^{\circ} \mathrm{C}$ in a humidified incubator with $5 \% \mathrm{CO}_{2}$, the non-invading cells that remained on the upper surface of the membrane were removed by gentle scraping. The invasive cells attached to the lower surface of the membrane insert were fixed in $10 \%$ formalin at room temperature for $30 \mathrm{~min}$ and stained with $0.05 \%$ crystal violet. The number of invasive cells on the lower surface of the membrane was then counted under a microscope.

Cell apoptosis assay. Cells transfected with si-ANRIL, or si-NC were harvested $48 \mathrm{~h}$ and then collected. After the double staining with FITC Annexin V and PI was done using the FITC Annexin $\mathrm{V}$ apoptosis detection kit (BD Biosciences) according to the manufacturer's protocol, the cells were analyzed with a flow cytometry (FACScan) equipped with a CellQuest software (both from BD Biosciences). Cells were discriminated into viable cells, dead cells, early apoptotic cells, and apoptotic cells, and then the relative ratio of early apoptotic cells was compared to control transfectant from each experiment.

Statistical analysis. All statistical analyses were performed by using SPSS 17.0 software (IBM, Chicago, IL, USA). The significance of differences between groups was estimated by the Student's t-test. Correlation between gene expressions were analyzed with regression analysis. Two-sided p-values were calculated, and differences were considered to be statistically significant at $\mathrm{P}<0.05$.

\section{Results}

ANRIL is upregulated in cisplatin-resistant and 5-FU-resistant gastric cancer tissues and cells. To investigate whether ANRIL participates in the development of MDR in gastric cancer tissue, we examined the mRNA levels of ANRIL in the gastric cancer tissues of cisplatin-sensitive patients $(n=36)$ and cisplatin-resistant patients $(n=11), 5-F U-s e n s i t i v e$ patients $(n=28)$ and 5-FU-resistant patients $(n=8)$. As a result, significantly elevated mRNA level of $A N R I L$ was observed in the cancer tissues of cisplatin-resistant or 5-FU-resistant patients comparing to drug-sensitive patients (Fig. 1A and B). To verify this differential expression of $A N R I L$, we detected ANRIL expression in cisplatin-resistant cells BGC823/DDP and 5-FU-resistant cells BGC823/5-FU, which were both developed from the parental BGC823 cells. Consistent with the results in gastric cancer tissues, ANRIL was greatly upregulated in BGC823/DDP and BGC823/5-FU cells (Fig. 1C). These results indicated that ANRIL may be associated with the development of cisplatin and 5-FU resistance in gastric cancer.

Knockdown of ANRIL inhibits gastric cancer cell proliferation in vitro. To investigate the potential role of ANRIL on gastric cancer cell proliferation, ANRIL siRNA was transfected into BGC823/DDP and BGC823/5-FU cells. To ensure the efficiency of interference and avoid off-target effects, qRT-PCR assays revealed that ANRIL expression was significantly reduced after transfection with si-ANRIL, while other lncRNAs, such as HOTAIR (17), PVT1 (18), and H19 (19) showed nearly no change (Fig. 2A and B). Then MTT assay showed that knockdown of ANRIL expression significantly inhibited cell proliferation both in BGC823/DDP and BGC823/5-FU cells compared with control cells (Fig. 2C and D).

Knockdown of ANRIL inhibits gastric cancer cell migration and invasion in vitro. Migration and invasion are significant aspect of cancer progression, which are involved in the dissolution of extracellular matrix proteins and the migration of tumor cells into contiguous tissues. To investigate whether ANRIL had a direct functional role in cell invasion in gastric cancer, we performed wound-healing assay and Transwell assay. The results showed that inhibition of ANRIL could significantly suppress BGC823/DDP and BGC823/5-FU cell migration (Fig. 3A and B) and invasion ability (Fig. 3C and D) when compared with control cells.

Knockdown of ANRIL promotes gastric cancer cell apoptosis in vitro. We next examined the effect of ANRIL expression on the apoptosis of GC cells. The results of flow cytometry revealed that BGC823/DDP (Fig. 4A and B) and BGC823/5-FU 

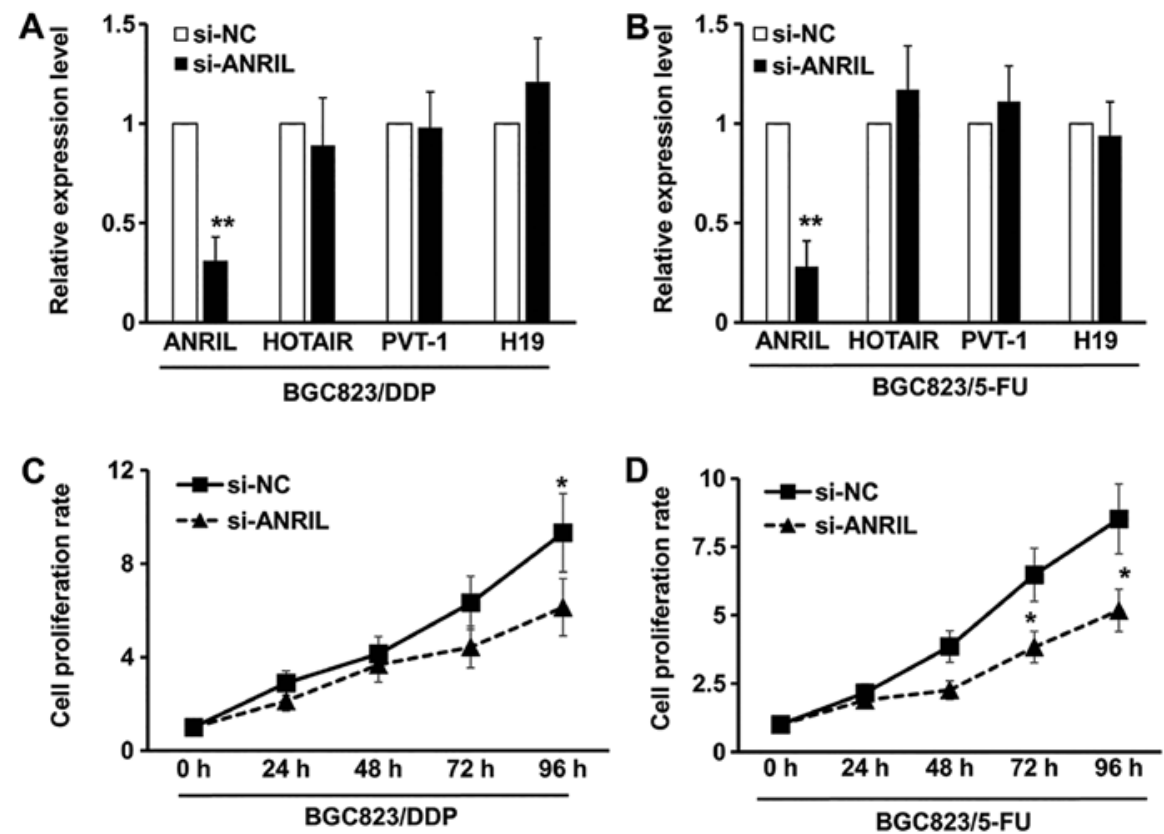

Figure 2. Knockdown of ANRIL inhibits gastric cancer cells proliferation in vitro. (A and B) The mRNA levels of four lncRNAs in the BGC823/DDP cells (A) or BGC823/5-FU cells (B) transfected with si-NC or si-ANRIL nucleotide fragment, including ANRIL, HOTAIR, PVT-1 and H19. (C and D) CCK-8 assays were performed to determine the cell viability and cell proliferation of BGC823/DDP cells (C) or BGC823/5-FU cells (D) transfected with si-NC or si-ANRIL nucleotide fragment. Error bars indicate means \pm standard errors of the mean. ${ }^{*} \mathrm{P}<0.05$ vs control.

A

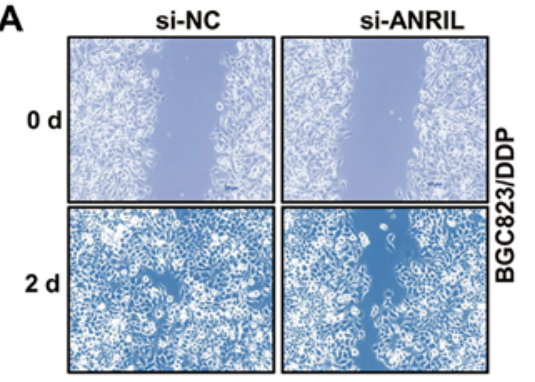

C

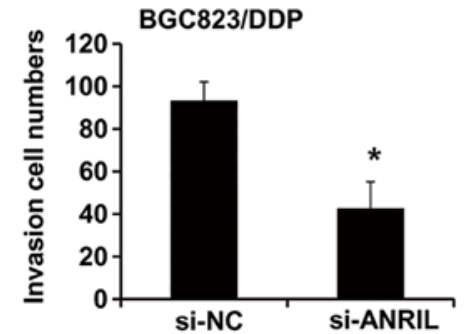

B

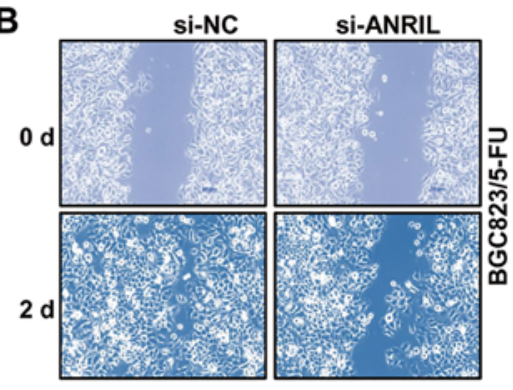

D

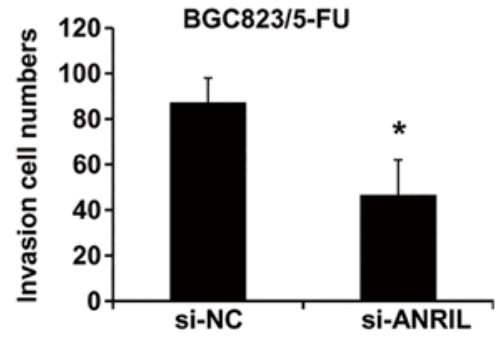

Figure 3. Knockdown of ANRIL inhibits gastric cancer cell migration and invasion in vitro. (A and B) Wound healing assay was performed to determine the cell migration ability. Representative images of wound healing assay of BGC823/DDP cells (A) or BGC823/5-FU cells (B) transfected with si-NC or si-ANRIL nucleotide fragment. Transwell assay was performed to determine the cell invasion ability. Invasion ability of BGC823/DDP (C) or BGC823/5-FU cells (D) transfected with si-NC or si-ANRIL nucleotide fragment. Error bars indicate means \pm standard errors of the mean. ${ }^{*} \mathrm{P}<0.05$ vs. control.

cells (Fig. 4C and D) transfected with si-ANRIL exhibited a significantly increased apoptosis index compared to the control groups $(\mathrm{P}<0.05)$. These data indicate that knockdown of ANRIL promotes cisplatin-induced apoptosis in BGC823/ DDP and 5-FU-induced apoptosis in BGC823/5-FU cells.

ANRIL knockdown reverses the drug resistance in cisplatinand 5-FU-resistant GC cell lines. On the basis of above results, we examined the effect of ANRIL knockdown on cisplatin- or 5-FU-induced cytotoxicity in BGC823/DDP and BGC823/5-FU cells. After transfected with si-ANRIL, BGC823/DDP and BGC823/5-FU cells were treated with cisplatin or 5-FU for $48 \mathrm{~h}$, and CCK-8 assay was performed to detect the cytotoxicity. As shown in Tables I and II, cells transfected with si-ANRIL had a significantly lower IC $_{50}$ values than that in si-NC group. These results indicate that si-ANRIL reverses the MDR in drug-resistant GC cell lines.

Knockdown of endogenous ANRIL downregulates MDR-related gene expression. To investigate the mechanisms 
A

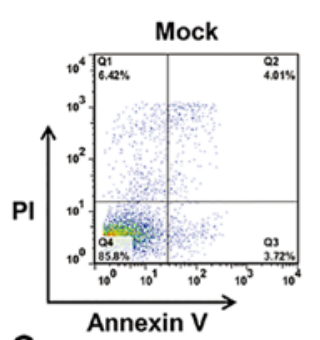

C

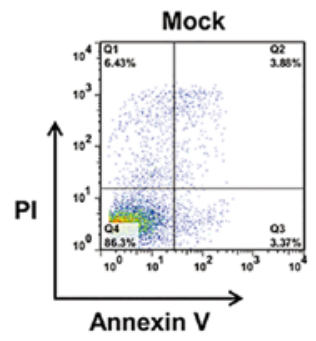

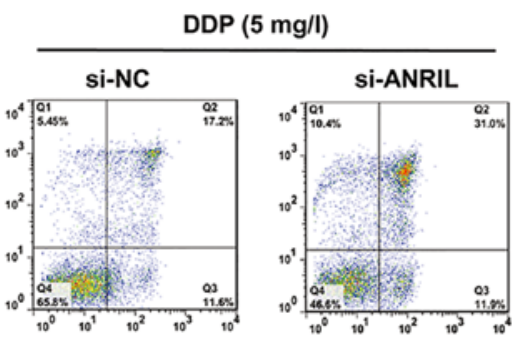

5-FU (100 mg/l)
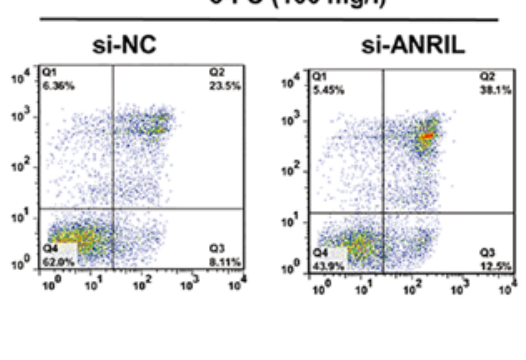

B

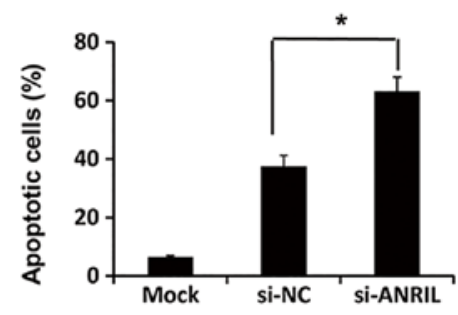

D

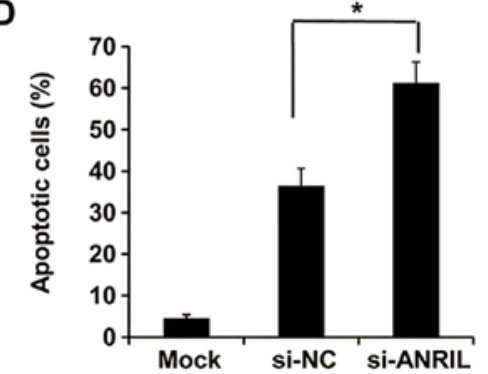

Figure 4. Knockdown of ANRIL promotes gastric cancer cells apoptosis in vitro. Flow cytometry analysis for the cell apoptosis in BGC823/DDP cells and BGC823/5-FU cells. After transfection with si-NC or si-ANRIL nucleotide fragment for $48 \mathrm{~h}, \mathrm{BGC} 823 / \mathrm{DDP}$ cells (A) or BGC823/5-FU cells (B) were treated with DDP or 5-FU and analyzed by flow cytometry. (C and D) Three independent experiments were summarized and the means and standard deviations were analyzed, respectively. "P<0.05 vs. control.

Table I. $\mathrm{IC}_{50}$ for cisplatin in different cells.

\begin{tabular}{llccc}
\hline Group & Cell lines & Treatment & $\mathrm{IC}_{50}(\mathrm{mg} / \mathrm{l})$ & Relative fold \\
\hline Group 1 & MGC823 & No & $0.67 \pm 0.14$ & $1.00 \pm 0.21$ \\
& MGC823/DDP & No & $9.5 \pm 2.6$ & $14.18 \pm 3.89^{\mathrm{a}}$ \\
Group 2 & MGC823/DDP & si-NC & $10.15 \pm 2.3$ & $15.14 \pm 3.43$ \\
& MGC823/DDP & si-ANRIL & $3.77 \pm 0.94$ & $5.63 \pm 1.40^{\mathrm{b}}$ \\
\hline
\end{tabular}

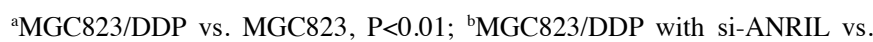
si-NC, $\mathrm{P}<0.05$.

Table II. $\mathrm{IC}_{50}$ for 5-FU in different cells.

\begin{tabular}{ccccc}
\hline Group & Cell lines & Treatment & $\mathrm{IC}_{50}(\mathrm{mg} / \mathrm{l})$ & Relative fold \\
\hline Group 3 & MGC823 & No & $5.73 \pm 0.85$ & $1.00 \pm 0.15$ \\
& MGC823/5-FU & No & $237.61 \pm 48.91$ & $41.47 \pm 8.54^{\mathrm{a}}$ \\
Group 4 & MGC823/5-FU & si-NC & $261.33 \pm 51.24$ & $45.61 \pm 8.94$ \\
& MGC823/5-FU & si-ANRIL & $57.88 \pm 9.45$ & $10.10 \pm 1.65^{\mathrm{b}}$ \\
\hline
\end{tabular}

${ }^{\mathrm{a} M G C 823 / 5-F U ~ v s . ~ M G C 823, ~ P<0.001 ; ~}{ }^{\mathrm{b}} \mathrm{MGC} 823 / 5-\mathrm{FU}$ with si-ANRIL vs. si-NC, $\mathrm{P}<0.05$.

underlying ANRIL induced MDR, we detected the expression of mRNA of several MDR-related genes by RT-PCR including $M D R 1$ and $M R P 1$ in different group. The levels of MDR1 and $M R P 1$ mRNA were both significantly decreased in BGC823/ DDP and BGC823/5-FU cells transfected with si-ANRIL compared with si-NC (Fig. 5A and B). Protein levels of MDR1 and MRP1 were also detected, and were increased in BGC823/ DDP and BGC823/5-FU cells transfected with si-ANRIL compared with si-NC (Fig. 5C). These results suggested that knockdown of endogenous ANRIL could downregulate the MDR-related gene expression in BGC823/DDP and BGC823/5-FU cells.

ANRIL expression positively correlates with the MDR-related gene expression. To investigate whether there is a correlation between the expression of ANRIL and the multidrug resistance gene $M D R I$ and $M R P 1$ in vivo, we isolated $\mathrm{mRNA}$ from the 64 gastric cancer tissues enrolled in this study, and analyzed the expressions of $A N R I L, M D R 1$, and $M R P 1$ using real-time PCR. After analyzed using the regression analysis, we found the R-square of $A N R I L$ and MDRI expression was 0.623 (Fig. 6A), and the R-square of ANRIL and MRPI expression was 0.612 (Fig. 6B), which indicated their goodness-of-fit of linear regression, and changes of ANRIL expression relate to changes of MDRI and MRPI $(\mathrm{P}<0.05$, respectively). These results strongly suggest that ANRIL may regulate the expression of $M D R 1$ and $M R P 1$ in gastric cancer patients.

\section{Discussion}

The development of MDR to cancer chemotherapy is a major obstacle to the effective treatment of gastric cancer (20). MDR plays a critical role in tumor initiation and progression by promoting cell proliferation and inhibiting apoptosis (21). Various mechanisms contribute to MDR, for example, MDR1 and MRP1, both of which belong to the ATP-binding cassette (ABC) superfamily, and play important roles in MDR development (22). These transporter proteins mediate the efflux of drugs in the MDR spectrum, out of cells and thus reduce drug efficacy. In this study, cisplatin and 5-FU are selected as the model anticancer drugs, because it is a substrate of MDR1 and MRP1 $(23,24)$.However, the mechanism of MDR remains unclear. 
A

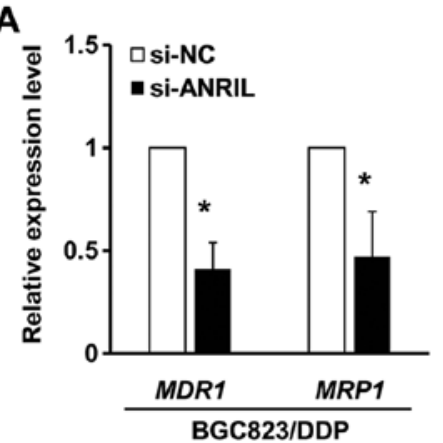

B

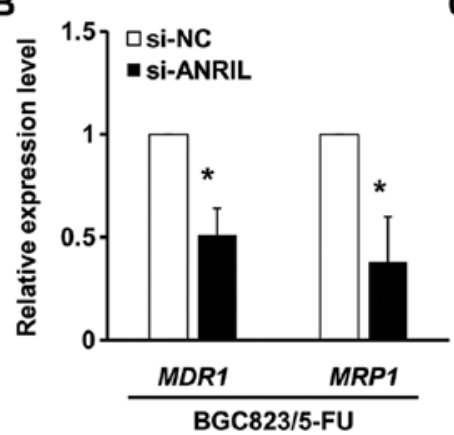

C
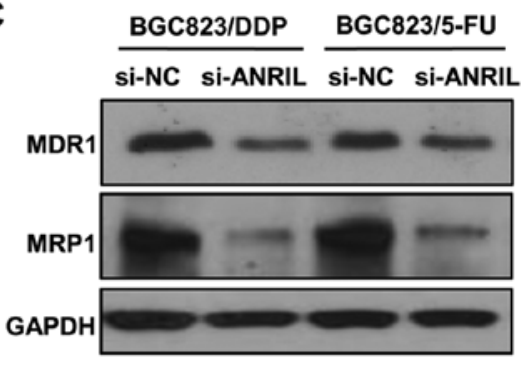

Figure 5. Knockdown of endogenous ANRIL downregulates MDR-related gene expression. (A and B) Quantitative RT-PCR assays were performed to detect the mRNA level of MDR1 and MRP1 in BGC823/DDP cells (A) or BGC823/5-FU cells (B) transfected with si-NC or si-ANRIL nucleotide fragment. (C) Western blot assays were performed to detect the protein level in BGC823/DDP cells or BGC823/5-FU cells transfected with si-NC or si-ANRIL nucleotide fragment. Error bars indicate means \pm standard errors of the mean. ${ }^{*} \mathrm{P}<0.05$ vs. control.

A

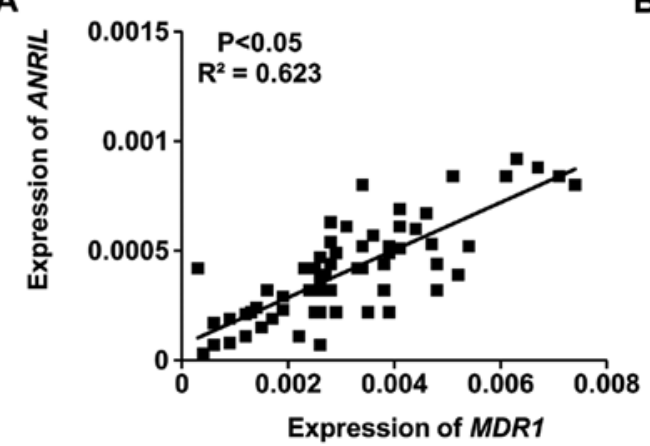

B

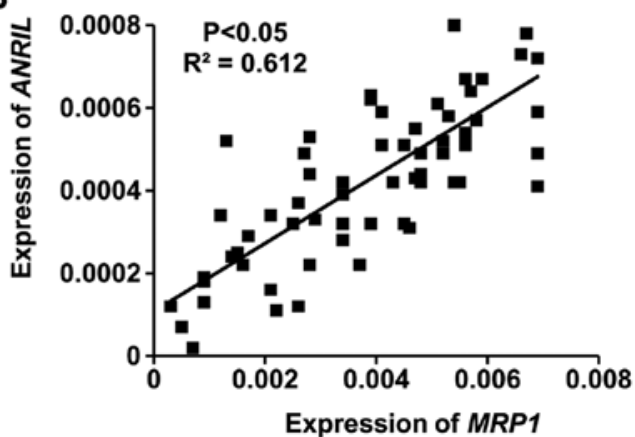

Figure 6. Positive correlations between ANRIL expression and the MDR-related genes. The regression analysis was performed between the ANRIL expression and $M D R 1$ expression (A), MRPl expression (B), and the R-square representing the goodness-of-fit.

Recent improvements in high-throughput gene expression analysis have led to the discovery that transcription from $<2 \%$ of the human genome yields many lncRNAs with limited or no protein-coding capacity because of a lack of open reading frames (25). Dysregulated expression of these lncRNAs have been revealed in several human diseases but their exact biological functions on MDR are unclear. Zhang et al showed that PVT-1 (plasmacytoma variant translocation 1) was highly expressed in gastric cancer tissues of cisplatin-resistant patients and cisplatin-resistant cells, and overexpression of PVT1 in gastric carcinoma promotes the development of MDR (26); Wang et al reported lncRNA MRUL (MDR-related and upregulated lncRNA) was significantly upregulated in two multidrug-resistant gastric cancer cell lines, and MRUL knockdown in these two cells led to increased rates of apoptosis, increased accumulation, and reduced doxorubicin release in the presence of adriamycin or vincristine (27); Jiang et al showed that a specific differentially expressed lncRNA ARA (adriamycin resistance associated) was validated in MCF-7/ADR and HepG2/ADR cells, ARA expression is significantly associated with adriamycin sensitivity in a panel of breast and liver cancer cell lines, and ARA knockdown reduced the proliferation, induced cell death, G2/M arrest and migration defects (28); Jin et al showed that lncRNA MEG3 (maternally expressed gene 3) was decreased in cisplatin-insensitive lung adeno- carcinoma tissues while p53 protein levels were decreased and Bcl-xl protein levels increased, MEG3 overexpression in A549/DDP cells increased their chemosensitivity to cisplatin both in vitro and in vivo by inhibiting cell proliferation and inducing apoptosis. By contrast, MEG3 knockdown in A549 cells decreased chemosensitivity (29). Taken together, lncRNA might participate in the process of MDR in gastric cancer.

ANRIL is transcribed in anti-sense direction with respect to the primary INK4 and ARF transcripts (12). ANRIL was shown to be involved in epigenetic regulation of the INK4BARF-INK4A locus by direct binding to the INK4b transcript and recruiting the Polycomb repressor complex (PRC) to repress the transcription of genes at this locus (30). Recent studies reported that ANRIL exerts its biological function in several kinds of cancer, such as hepatocellular carcinoma, lung cancer, ovarian cancer and gastric cancer. Qiu et al reported that ANRIL was highly expressed in ovarian cancer cells, siRNA-mediated ANRIL silencing in ovarian cancer cells impaired cell migration and invasion (31); Lin et al showed that higher expression of ANRIL in non-small cell lung cancer (NSCLC) tissues was associated with higher TNM stage and advanced lymph node metastasis, moreover, knockdown of ANRIL expression inhibited NSCLC cell proliferation, migration and invasion in vitro (32); Huang et al showed that ANRIL expression was 
upregulated in hepatocellular carcinoma (HCC) tissues, and the higher expression of ANRIL was significantly correlated with tumor size and Barcelona Clinic Liver Cancer (BCLC) stage, knockdown of ANRIL expression impaired cell proliferation and invasion and induced cell apoptosis both in vitro and in vivo. For the detailed mechanism, ANRIL was able to epigenetically repress KLF2 transcription in HCC cells by binding with PRC2 and recruiting it to KLF2 promoter region (33). In respect of gastric cancer, Zhang et al showed that in a cohort of 120 gastric cancer patients, the higher expression of ANRIL was significantly correlated with a higher TNM stage and tumor size, and ANRIL knockdown could significantly repress the proliferation both in vitro and in vivo. In addition, E2F1 could induce ANRIL and ANRIL-mediated growth promotion in part due to epigenetic repression of $\mathrm{miR}-99 \mathrm{a} / \mathrm{miR}-449 \mathrm{a}$ in Trans (controlling the targets - mTOR and CDK6/E2F1 pathway) by binding to PRC2, thus forming a positive feedback loop, continuing to promote gastric cancer cell proliferation (34). Though there are several papers on the exploration and the function of ANRIL, the effect of ANRIL on the MDR in gastric cancer is still unclear.

In summary, the expression of ANRIL was significantly upregulated in cisplatin-resistance and 5-FU-resistance gastric cancer tissues and cells, suggesting that its overexpression may be an important factor for gastric cancer MDR. We showed that silencing ANRIL may inhibit the proliferation and invasion ability, and promote the apoptosis rate of drug-resistance gastric cancer cells, which suggested that ANRIL contributes to gastric cancer cell biological function. Further insights into the functional implications of ANRIL and its downstream genes related with MDR, which are identified as MDR1 and MRP1, may contribute to the understanding of gastric cancer MDR, and facilitate the development of ANRIL therapeutics against gastric cancer.

\section{References}

1. Jemal A, Bray F, Center MM, Ferlay J, Ward E and Forman D Global cancer statistics. CA Cancer J Clin 61: 69-90, 2011.

2. Yang T, Kong B, Kuang Y, Cheng L, Gu J, Zhang J, Shu H, Yu S, Yang $\mathrm{X}$, Cheng $\mathrm{J}$, et al: Emodin plays an interventional role in epileptic rats via multidrug resistance gene 1 (MDR1). Int J Clin Exp Pathol 8: 3418-3425, 2015.

3. Ma H, Cheng L, Hao K, Li Y, Song X, Zhou H and Jia L: Reversal effect of ST6GAL 1 on multidrug resistance in human leukemia by regulating the PI3K/Akt pathway and the expression of P-gp and MRP1. PLoS One 9: e85113, 2014.

4. Zheng Z, Liu B and Wu X: RhoGDI2 up-regulates P-glycoprotein expression via Rac1 in gastric cancer cells. Cancer Cell Int 15: 41, 2015.

5. Zhao J, Liu Y, Huang G, Cui P, Zhang W and Zhang Y: Long non-coding RNAs in gastric cancer: Versatile mechanisms and potential for clinical translation. Am J Cancer Res 5: 907-927, 2015.

6. Du Z, Fei T, Verhaak RG, Su Z, Zhang Y, Brown M, Chen Y and Liu XS: Integrative genomic analyses reveal clinically relevant long noncoding RNAs in human cancer. Nat Struct Mol Biol 20: 908-913, 2013

7. Autuoro JM, Pirnie SP and Carmichael GG: Long noncoding RNAs in imprinting and $\mathrm{X}$ chromosome inactivation Biomolecules 4: 76-100, 2014.

8. Ghosal S, Das S and Chakrabarti J: Long noncoding RNAs: New players in the molecular mechanism for maintenance and differentiation of pluripotent stem cells. Stem Cells Dev 22: 2240-2253, 2013.
9. Ounzain S, Pezzuto I, Micheletti R, Burdet F, Sheta R, Nemir M, Gonzales C, Sarre A, Alexanian M, Blow MJ, et al: Functional importance of cardiac enhancer-associated noncoding RNAs in heart development and disease. J Mol Cell Cardiol 76: 55-70, 2014.

10. Serviss JT, Johnsson P and Grandér D: An emerging role for long non-coding RNAs in cancer metastasis. Front Genet 5: 234, 2014.

11. PopovNandGilJ: Epigenetic regulation of theINK4b-ARF-INK4a locus: In sickness and in health. Epigenetics 5: 685-690, 2010.

12. Pasmant E, Laurendeau I, Héron D, Vidaud M, Vidaud D and Bièche I: Characterization of a germ-line deletion, including the entire INK4/ARF locus, in a melanoma-neural system tumor family: Identification of ANRIL, an antisense noncoding RNA whose expression coclusters with ARF. Cancer Res 67: 3963-3969, 2007

13. Yap KL, Li S, Muñoz-Cabello AM, Raguz S, Zeng L, Mujtaba S, Gil J, Walsh MJ and Zhou MM: Molecular interplay of the noncoding RNA ANRIL and methylated histone H3 lysine 27 by polycomb CBX7 in transcriptional silencing of INK4a. Mol Cell 38: 662-674, 2010.

14. Aguilo F, Zhou MM and Walsh MJ: Long noncoding RNA, polycomb, and the ghosts haunting INK4b-ARF-INK4a expression. Cancer Res 71: 5365-5369, 2011.

15. Pasmant E, Sabbagh A, Masliah-Planchon J, Ortonne N, Laurendeau I, Melin L, Ferkal S, Hernandez L, Leroy K, Valeyrie-Allanore L, et al; NF France Network: Role of noncoding RNA ANRIL in genesis of plexiform neurofibromas in neurofibromatosis type 1. J Natl Cancer Inst 103: 1713-1722, 2011.

16. Özgür E, Mert U, Isin M, Okutan M, Dalay N and Gezer U: Differential expression of long non-coding RNAs during genotoxic stress-induced apoptosis in HeLa and MCF-7 cells. Clin Exp Med 13: 119-126, 2013.

17. Zhang ZZ, Shen ZY, Shen YY, Zhao EH, Wang M, Wang CJ, $\mathrm{Cao} \mathrm{H}$ and $\mathrm{Xu}$ J: HOTAIR long noncoding RNA promotes gastric cancer metastasis through suppression of poly $\mathrm{r}(\mathrm{C})$-binding protein (PCBP) 1. Mol Cancer Ther 14: 1162-1170, 2015.

18. Kong R, Zhang EB, Yin DD, You LH, Xu TP, Chen WM, Xia R, Wan L, Sun M, Wang ZX, et al: Long noncoding RNA PVT1 indicates a poor prognosis of gastric cancer and promotes cell proliferation through epigenetically regulating $\mathrm{p} 15$ and $\mathrm{p} 16 . \mathrm{Mol}$ Cancer 14: 82, 2015.

19. Li H, Yu B, Li J, Su L, Yan M, Zhu Z and Liu B: Overexpression of lncRNA H19 enhances carcinogenesis and metastasis of gastric cancer. Oncotarget 5: 2318-2329, 2014.

20. Verstraelen J and Reichl S: Multidrug resistance-associated protein (MRP1, 2, 4 and 5) expression in human corneal cell culture models and animal corneal tissue. Mol Pharm 11: 2160-2171, 2014.

21. He T, Mo A, Zhang K and Liu L: ABCB1/MDR1 gene polymorphism and colorectal cancer risk: A meta-analysis of case-control studies. Colorectal Dis 15: 12-18, 2013.

22. Lo YL and Liu Y: Reversing multidrug resistance in Caco-2 by silencing MDR1, MRP1, MRP2, and BCL-2/BCL-xL using liposomal antisense oligonucleotides. PLoS One 9: e90180, 2014.

23. Wang TH, Wan JY, Gong X, Li HZ and Cheng Y: Tetrandrine enhances cytotoxicity of cisplatin in human drug-resistant esophageal squamous carcinoma cells by inhibition of multidrug resistance-associated protein 1. Oncol Rep 28: 1681-1686, 2012.

24. Zheng G, Peng F, Ding R, Yu Y, Ouyang Y, Chen Z, Xiao Z and $\mathrm{He} Z$ : Identification of proteins responsible for the multiple drug resistance in 5-fluorouracil-induced breast cancer cell using proteomics analysis. J Cancer Res Clin Oncol 136: 1477-1488, 2010.

25. Gibb EA, Brown CJ and Lam WL: The functional role of long non-coding RNA in human carcinomas. Mol Cancer 10: 38, 2011.

26. Zhang XW, Bu P, Liu L, Zhang XZ and Li J: Overexpression of long non-coding RNA PVT1 in gastric cancer cells promotes the development of multidrug resistance. Biochem Biophys Res Commun 462: 227-232, 2015.

27. Wang Y, Zhang D, Wu K, Zhao Q, Nie Y and Fan D: Long noncoding RNA MRUL promotes ABCB1 expression in multidrug-resistant gastric cancer cell sublines. Mol Cell Biol 34: 3182-3193, 2014.

28. Jiang M, Huang O, Xie Z, Wu S, Zhang X, Shen A, Liu H, Chen X, Wu J, Lou Y, et al: A novel long non-coding RNA-ARA: Adriamycin resistance-associated. Biochem Pharmacol 87: 254-283, 2014. 
29. Liu J, Wan L, Lu K, Sun M, Pan X, Zhang P, Lu B, Liu G and Wang Z: The long noncoding RNA MEG3 contributes to cisplatin resistance of human lung adenocarcinoma. PLoS One 10: e0114586, 2015.

30. Kotake Y, Nakagawa T, Kitagawa K, Suzuki S, Liu N, Kitagawa M and Xiong Y: Long non-coding RNA ANRIL is required for the PRC2 recruitment to and silencing of p15(INK4B) tumor suppressor gene. Oncogene 30: 1956-1962, 2011.

31. Qiu JJ, Lin YY, Ding JX, Feng WW, Jin HY and Hua KQ: Long non-coding RNA ANRIL predicts poor prognosis and promotes invasion/metastasis in serous ovarian cancer. Int J Oncol 46: 2497-2505, 2015.

32. Lin L, Gu ZT, Chen WH and Cao KJ: Increased expression of the long non-coding RNA ANRIL promotes lung cancer cell metastasis and correlates with poor prognosis. Diagn Pathol 10: $14,2015$.
33. Huang MD, Chen WM, Qi FZ, Xia R, Sun M, Xu TP, Yin L, Zhang EB, De W and Shu YQ: Long non-coding RNA ANRIL is upregulated in hepatocellular carcinoma and regulates cell apoptosis by epigenetic silencing of KLF2. J Hematol Oncol 8: $50,2015$.

34. Zhang EB, Kong R, Yin DD, You LH, Sun M, Han L, Xu TP, Xia R, Yang JS, De W, et al: Long noncoding RNA ANRIL indicates a poor prognosis of gastric cancer and promotes tumor growth by epigenetically silencing of miR-99a/miR-449a. Oncotarget 5: 2276-2292, 2014. 